\title{
Materials studies by the bilbao crystallographic server
}

Mois Ilia Aroyo $^{1}$, J. Manuel Perez-Mato ${ }^{1}$, Luis Elcoro ${ }^{1}$, Gotzon Madariaga ${ }^{1}$, Samuel Vidal Gallego ${ }^{1}$, Gemma de la Flor Martin ${ }^{1}$, Emre S. Tasci $^{2}$

${ }^{1}$ Condensed-Matter Physics Department, University Of The Basque Country (UPV/EHU), Bilbao, Spain, ${ }^{2}$ Department of Physics Engineering, Hacettepe University, Ankara, Turkey

E-mail: mois.aroyo@ehu.es

The Bilbao Crystallographic Server (www.cryst.ehu.es) is a free web site with an access to crystallographic data of space and point groups, magnetic space groups, subperiodic groups, their representations and group-subgroup relations [1]. Wide range of complex solid-state physics and structure-chemistry aspects of materials studies are facilitated by the specialized software provided by the server.

The server offers a set of structure-utility programs including basic tools for transformations between different structure descriptions or transformations compatible with a specific symmetry reduction. There is an online tool (COMPSTRU) for a quantitative analysis of the similarity of two structure models, also helpful for the recognition of identical or nearly identical atomic arrangements of different compounds. The program STRUCTURE RELATIONS for the analysis of structure relations between two phases of the same compound with group-subgroup related space groups is of great utility for the construction of family trees of homeotypic crystal structures, known as Baernighausen trees. The program AMPLIMODES performs the decomposition of the global distortion into symmetry-mode contributions and classifies the correlated atomic displacements separating the so-called primary modes (fundamental for the phase stability), from the weaker distortions of limited relevance for the transition mechanism [2]. The server also offers online tools for the evaluation of the pseudosymmetry of a given structure with respect to a supergroup of its space group, which could serve as a powerful method for the prediction of new ferroic materials. Of special interest is the program SUBGROUPS which extends further the capabilities of the server in the symmetry characterization of distorted structures by providing their possible subgroup symmetries given the relationship of the distorted to the parent undistorted lattice.

Recently implemented computational tools and databases in the server allow the systematic application of symmetry arguments in the study of magnetic structures [3]. There is an online access to basic crystallographic data of magnetic space groups in different settings (MGENPOS, MWYCKPOS), to the systematic absences for non-polarized neutron magnetic diffraction and also to the symmetry-adapted forms of the corresponding structure factors (MAGNEXT). The user can identify a magnetic space group from its symmetry operations given in an arbitrary setting (IDENTIFY MAGNETIC GROUP), derive the possible magnetic space groups for a given set of propagation vectors (MAXMAGN, k-SUBGROUPSMAG) or generate a magnetic structure model complying with a chosen magnetic space group (MAGMODELIZE). The server offers an access to a database of more than 400 published magnetic structures (MAGNDATA) described using magnetic space groups for commensurate structures, and magnetic superspace groups for incommensurate structures.

The presentation of the databases and programs offered by the Bilbao Crystallographic Server will be accompanied by case studies illustrating the capacity and efficiency of the online tools in material studies.

[1] Aroyo M.I. et al. (2006) Z. Kristallogr. 221, 15-27.

[2] Perez-Mato J.M. et al. (2010) Acta Cryst A66, 558-590.

[3] Perez-Mato J.M. et al. (2015) Annu. Rev. Mater. Res. 45:13.1-13.32.

Keywords: Bilbao Crystallographic Server, structural phase transitions, magnetic crystallography 